\title{
Penetrating injuries due to gunshot wounds involving the brachial plexus
}

\author{
Daniel H. Kim, M.D., Judith A. Murovic, M.D., Robert L. Tiel, M.D., \\ AND DAVID G. KLINE, M.D. \\ Department of Neurosurgery, Stanford University Medical Center, Stanford, California; \\ and Department of Neurosurgery, Louisiana State University Health Sciences Center, \\ New Orleans, Louisiana
}

\begin{abstract}
The authors review 118 operative brachial plexus gunshot wounds (GSWs), causing 293 element injuries that were managed over a 30-year period at Louisiana State University Health Sciences Center (LSUHSC). Retrospective chart reviews were performed. Using the LSUHSC grading system for motor sensory function, each element's grades were combined and averaged.

Most of the 293 injured elements were found to have gross continuity at operation and of 202 elements with complete neurological loss, only $16(8 \%)$ exhibited total disruption. Of 293 injuries, 128 elements with complete or incomplete loss were not only in continuity when explored but also had positive intraoperative nerve action potentials (NAPs). After neurolysis, 120 of 128 elements in continuity (94\%) improved to greater than or equal to Grade 3 function. Elements not regenerating early usually required repair. One hundred fifty-six of 202 completely or incompletely injured elements $(77 \%)$ required resection and suture or graft repair based on intraoperative NAPs. Neurolysis achieved greater than or equal to Grade 3 results in $42(91 \%)$ of 46 elements with complete loss. Suture repair resulted in good outcomes in 14 (67\%) of 21 and in $73(54 \%)$ of 135 undergoing graft repairs (1 to $3.5 \mathrm{~cm}$ length) and presenting with complete loss.

Of 91 incomplete elements, intraoperative NAPs were positive in $82(90 \%)$ and 78 of 82 had good results. Nine had negative NAPs and six elements required suture repair. Three required grafts with results of greater than or equal to Grade 3 in five $(83 \%)$ of six and two $(67 \%)$ of three, respectively.

Based on 118 patient results with 293 injured elements, guidelines for the management of GSWs were established as described in this paper.
\end{abstract}

\section{KEY WORDS • brachial plexus injury • anastomosis • nerve graft • nerve action potential}

In this paper, we report on the management of 118 patients with brachial plexus due to GSWs that resulted in 293 injured plexus elements. These patients underwent operations over a 30-year period at LSUHSC. Retrospective chart reviews provided data that were used to establish guidelines for the evaluation of patients with this injury and their selection for surgery. Associated vascular injuries, operative techniques, and functional outcomes are also addressed.

Overall, BPIs are uncommon lesions. In a report by Midha ${ }^{9}$ only 54 BPIs (1.2\%) were found in 4538 patients presenting to a regional trauma facility. A 30-year review from LSUHSC in 2003 by Kim, et al., ${ }^{4}$ however, documented 1019 operative brachial plexus lesions, of which 118 were due to GSWs (12\%). These GSWs were exceeded in number only by 509 lesions due to stretches/contu-

Abbreviations used in this paper: $\mathrm{BPI}=$ brachial plexus injury; CRPS = complex regional pain syndrome; GSW = gunshot wound; LSUHSC = Louisiana State University Health Sciences Center; $\mathrm{NAP}=$ nerve action potential. sion injuries (49\%). Peach and colleagues ${ }^{11}$ analyzed 218 BPIs and found a similar incidence of GSWs involving the brachial plexus. In their review, 42 GSWs involving the brachial plexus (19\%) were exceeded only by 111 traction injuries $(51 \%)$. These latter two reviews represent two of the largest in the literature. The paper by Kim, et al., can thus be used to provide guidelines to patient care.

Caruso, et al., ${ }^{1}$ reported on a trend toward the use of larger-caliber and thus more damage-inflicting firearms in accidents, homicides, and suicides in a study of 745 bullets from GSWs over 16 years between 1981 and 1997 at a Level I trauma center in Newark, New Jersey. Their study portends that GSWs of the brachial plexus, when they do occur, will be of even greater severity and have more devastating associated injuries to manage.

\section{CLINICAL MATERIAL AND METHODS}

Between 1968 and 1998, 118 patients underwent operations at LSUHSC for GSWs involving the brachial plexus and resulting in injury to 293 plexus elements. Patient 
charts were reviewed retrospectively to determine the type of injury, prior treatment, and preoperative neurological presentation. Electrophysiological studies were conducted preoperatively 3 to 4 weeks postinjury and these along with details of the surgical procedure performed at LSUHSC, intraoperative NAP recordings, pathological findings, associated vascular injuries, and postoperative functional status were recorded. The minimum follow-up period was 18 months with a mean of 42 months.

In this series of GSWs associated with the brachial plexus, pre- and postoperative neural functions were given a grade of 0 to 5 for each individual plexal element and the grades for the elements involved were combined and averaged. The LSUHSC grading system for grading preoperative motor and sensory function as well as level of recovery was used (Table 1). ${ }^{6}$ Successful outcomes were defined as a Grade 3 or higher, and good and excellent recoveries were represented by Grades 4 and 5, respectively.

The selective criteria used for neural operation (Table 2) included loss of function that was complete and persistent in the distribution of one or more of the elements with no improvement detected clinically or by electromyography in the early months following injury. Neural operation was reserved for complete loss in the distribution of at least one element usually improved by surgery, such as C-5, C6, C-7, upper or middle trunk, or the lateral or posterior cords or their outflows. Patients with injuries who suffered loss in the lower element(s) were not usually candidates for surgery. Other indications for neural operation were incomplete loss of function attended by non-CRPS not alleviated pharmacologically, pseudoaneurysm, clot or fistula compressing or involving the plexus, and true CRPS requiring sympathectomy.

Because most GSWs involving the plexus do not transect elements, but rather contuse, bruise, or stretch them, the majority of GSW plexus cases were managed conservatively for 2 to 5 months. A thorough baseline clinical examination was considered to be essential. Patients were then reevaluated on at least one occasion in subsequent months to establish if there were clinical signs of reversal of neurological loss or electrical evidence of reinner- vation. The average injury-to-operative interval was 17 weeks. Because many patients came from states other than Louisiana and from other countries for their initial evaluations and subsequent operations, these individuals could not always be scheduled at the optimal interval of 2 to 4 months between injury and operation. Again the usual reason for neural operation was loss in the distribution of one or more plexus elements that had failed to improve in the early months after injury.

\section{Operative Techniques}

Operative techniques for approaching the plexus have been detailed elsewhere ${ }^{6,7}$ and will not be repeated here. Only a few patients with GSWs involving the lower spinal nerves and/or proximal spinal nerves in or close to the foraminal level were considered candidates for the posterior approach. ${ }^{8}$

In this series, the supraclavicular GSW injuries were easier to approach technically than the infraclavicular lesions, where a length of the axillary and sometimes brachial artery with some of the axillary venous pathways needed to be freed and protected. Infraclavicular dissection was often made difficult by heavy scar tissue as well as vascular grafts from prior vascular repairs. Subclavian-axillary grafts associated with a mainly supraclavicular plexus injury were easier to manage because the repaired vascular segment tended to be inferomedial to the plexus elements being evaluated and repaired. Vascular repairs at an infraclavicular and/or axillary locus were usually at the epicenter of the injured neural elements. These neural elements were generally adherent to the vascular repair and at times had been sutured to vessels to help secure the repair. An essential step was to lay out the two major vessels, that is, artery and vein, and to repair any tears made during the dissection. Proximal and distal arterial control was obtained by fine rubber loops or vascular clamps. The profundus artery was spared, when possible, especially if the original injury or prior vascular graft repair had resulted in a more distal stenosis or thrombosis of the brachial artery.

Loupes of 3.5-power magnification were used for all

TABLE 1

Louisiana State University Health Sciences Center grading system by element of brachial plexus

\begin{tabular}{|c|c|c|}
\hline Grade & Evaluation & Description \\
\hline \multicolumn{3}{|c|}{ overall grade } \\
\hline 0 & absent & no muscle contraction \\
\hline 1 & poor & proximal muscles contract, but not against gravity \\
\hline 2 & fair & proximal muscles contract against gravity \& distal muscles do not contract; sensory grade, if applicable, is usually $<2$ \\
\hline 3 & moderate & $\begin{array}{l}\text { proximal muscles contract against gravity \& some resistance; some distal muscles contact against gravity; sensory grade, } \\
\text { if applicable, is usually } 3\end{array}$ \\
\hline 4 & good & all muscles contract against gravity \& some resistance; sensory grade, if applicable, is 3 or 4 \\
\hline 5 & excellent & all muscles contract against moderate resistance; sensory grade, if applicable, is 4 \\
\hline \multicolumn{3}{|c|}{ sensory grading* } \\
\hline 0 & absent & no response to touch, pin, or pressure \\
\hline 1 & poor & testing produces hyperesthesias or paresthesias; deep pain recovery in autonomous zones \\
\hline 2 & fair & sensory response sufficient for grip \& slow protection, sensory stimuli mislocalized w/ some overresponse \\
\hline 3 & moderate & response to touch \& pin in autonomous zones, sensation mislocalized \& not normal, w/ overresponse \\
\hline 4 & good & response to touch \& pin in autonomous zones, response localized but not normal; there is no overresponse \\
\hline 5 & excellent & response to touch \& pin in entire field of plexus element including autonomous zones \\
\hline
\end{tabular}

* Where applicable, such as at C-8, T-1, lower trunk, lateral cord, and medial cord. Adapted from J Neurosurg 98:1005-1016, 2003. 
TABLE 2

Gunshot wounds: criteria for selection for a neural operation

\begin{abstract}
complete loss in the distribution of at least one element and:
no improvement clinically or by EMG in early months after injury;

loss in the distribution of at least one element usually helped by operation: C-5, C-6, C-7, upper or middle trunk, lat or posterior cords or their outflows;

surgery was not performed for injuries w/ loss in lower element(s) only. If there was loss in other elements likely to be helped by operation then the lower elements were also inspected, evaluated electrically, and if possible repaired

incomplete loss where pain could not be controlled pharmacologically. In addition, when completely injured elements underwent surgery, those elements incompletely injured were dissected out and also checked by NAP recordings

pseudoaneurysm, clot, or fistula involving plexus

true causalgia requiring sympathectomy
\end{abstract}

dissections. The exposure of injured plexus elements was performed with a No. 15 blade on a plastic knife handle or a long pair of Metzenbaum scissors. Neural structures proximal and distal to the injury site were exposed whenever possible to aid in the approach to the injury site and to permit electrode placement for operative electrophysiological studies. In patients with shotgun or high-power rifle wounds and/or extensive prior surgery for vascular repair a long up-and-down exposure was necessary.

\section{Intraoperative NAP Studies}

Intraoperative stimulation and NAP recording studies were important for the identification of elements requiring or not requiring resection and were performed in each patient with results noted. If a NAP could be evoked through the injury site and conducted into the distal stump of the injured element, that particular element was spared resection. If the entire cross-section of the element appeared damaged and a NAP was absent, resection of that entire segment was indicated. When a portion of the crosssectional area of injury appeared less damaged than the remainder and exhibited a NAP, these elements, and there were several in this series, were treated with a split repair. The portion conducting a NAP was spared resection and the rest of the element at that level was repaired, usually by short 1- to 1.5-in grafts. Each injured segment that was resected because of the absence of a NAP was subsequently examined histologically and proved to be neurotmetic (Sunderland Grade IV).

When grafts were indicated, the proximal and distal stumps of the neural elements were split into fascicles or bundles of fascicles 1 to $2 \mathrm{~cm}$ long. Sural and/or sometimes antebrachial cutaneous grafts were secured proximally and distally using 7-0 or 8-0 sutures. Grafts were placed from a proximal group of fascicles to a distal group and thus formed an interfascicular graft repair.

\section{RESULTS}

There were 118 LSUHSC GSWs involving the brachial plexus that resulted in 293 injured plexus elements (Table 3). Most wounds in this civilian series were from bullets (Fig. 1), but some were from shell fragments, and all were caused by handguns, shotguns, or rifles. Approximately half of the wounds were associated with crimes and the rest were related to hunting accidents or, more frequently, to poor handling of weapons. Approximately one sixth of these wounds were inflicted during unsuccessful suicide attempts.
Approximately $40 \%$ of patients with lesions in continuity recovered spontaneously, but the other $60 \%$ or so did not display signs of reversal or reinnervation after several months. In these latter cases, exploration and intraoperative NAP recording were performed.

Of 293 operative plexus element lesions, 202 elements $(69 \%)$ had complete loss of function preoperatively both on neurological examination and preoperative electromyography. The remaining 91 elements (31\%) presented with incomplete loss based on results of these modalities.

Most of the 293 injured plexus elements had some degree of gross continuity when surgically exposed (Fig. 2). Of the 202 elements with complete neurological loss distal to the lesion, only $16(8 \%)$ were found to have total physical disruption during surgical exploration.

Of 293 GSWs involving brachial plexus elements, there were 128 elements presenting with either complete or incomplete loss that were found to be in continuity when explored. On intraoperative NAP testing these 128 elements had recordable NAPs. In response to neurolysis, $120(94 \%)$ of these 128 elements in continuity had their function improved postoperatively to Grade 3 or better.

Elements that did not show early evidence of regeneration usually required repair. Thus, 156 of the 202 completely or incompletely injured elements $(77 \%)$ required resection and repair by either suture or graft as indicated by intraoperative NAP recording. Pathological examination of resected specimens confirmed neurotmesis or Grade IV injuries based on the Sunderland classification.

Repairs were relatively effective for elements such as the upper trunk and C-5 and C-6 nerve roots and for lateral and posterior cords and their outflows. Acceptable results were achieved by neurolysis in 42 (91\%) of 46 elements presenting with complete loss of function. Good

TABLE 3

Outcomes of surgery for 118 GSW injuries*

\begin{tabular}{|c|c|c|c|c|}
\hline Type of Lesion & $\begin{array}{c}\text { No. of } \\
\text { Elements }\end{array}$ & Neurolysis & Suture & Graft \\
\hline $\begin{array}{l}\text { lesions w/ } \\
\text { complete loss }\end{array}$ & 202 & $\begin{array}{l}46 / 42 \\
(91 \%)\end{array}$ & $\begin{array}{l}21 / 14 \\
(67 \%)\end{array}$ & $\begin{array}{l}135 / 73 \\
(54 \%)\end{array}$ \\
\hline $\begin{array}{l}\text { lesions w/ } \\
\text { incomplete loss }\end{array}$ & 91 & $\begin{array}{l}82 / 78 \\
(95 \%)\end{array}$ & $\begin{array}{c}6 / 5 \\
(83 \%)\end{array}$ & $\begin{array}{c}3 / 2 \\
(67 \%)\end{array}$ \\
\hline total & 293 & $\begin{array}{c}128 / 120 \\
(94 \%)\end{array}$ & $\begin{array}{l}27 / 19 \\
(70 \%)\end{array}$ & $\begin{array}{l}138 / 75 \\
(54 \%)\end{array}$ \\
\hline
\end{tabular}

* Results are given as the total number of elements/the number of elements recovering to Grade 3 or higher. Reprinted from J Neurosurg 98:1005-1016, 2003. 


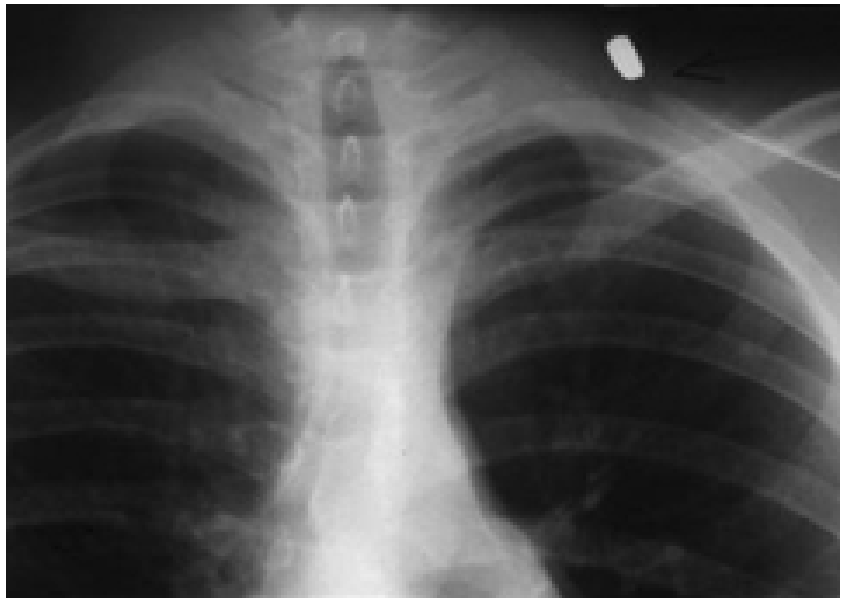

Fig. 1. A chest $\mathrm{x}$-ray film revealing a bullet lodged in the supraclavicular region that resulted in a severe brachial plexus palsy.

results were obtained by suture repair in $14(67 \%)$ of 21 cases and in $73(54 \%)$ of 135 graft repair cases both presenting with complete loss of function. Grafts worked as well as end-to-end suture repairs in the LSUHSC GSW series, perhaps because they were relatively short (1 to 2.5 in long; Fig. 3). Recovery occurred in severe C-8, T-1, lower trunk, and medial cord injuries when the nerve was in continuity and intraoperative NAPs were recordable. Graft or suture repair of these elements did not result in useful recovery, except in a few pediatric cases.

Of 91 elements preoperatively assessed as having incomplete loss, intraoperative stimulation and NAP studies were positive in the majority, that is, or $82(90 \%)$ of 91 elements and $78(95 \%)$ of these 82 had good results. Nine had negative NAP tests and were not regenerating adequately. These lesions required repair by suture in six ele-

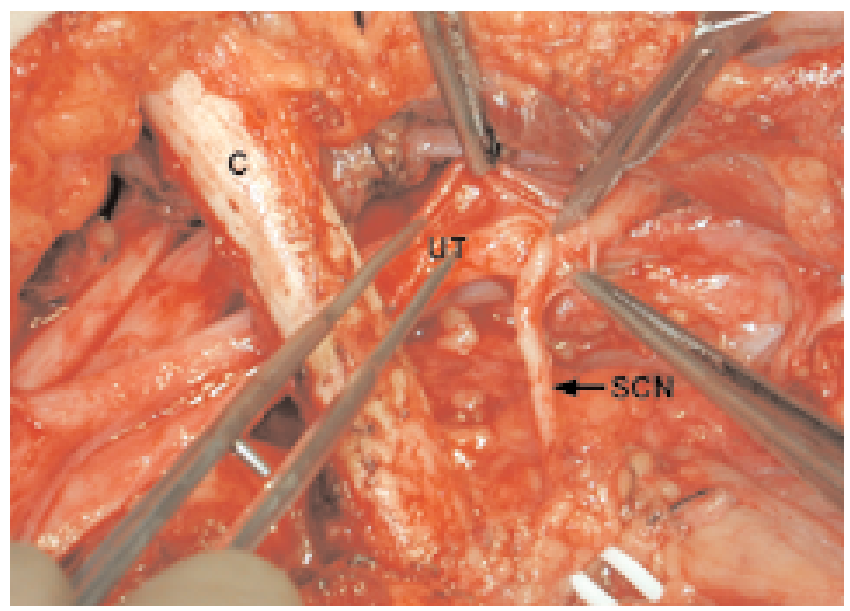

Fig. 2. An operative photograph of the left brachial plexus with the supraclavicular plexus to the right of the clavicle (C). A contusive injury occurred due to a GSW involving the upper trunk-tosuprascapular nerve. The suprascapular nerve was split away and spared because of a positive intraoperative NAP. UT = upper trunk; arrow depicts the suprascapular nerve $(\mathrm{SCN})$.

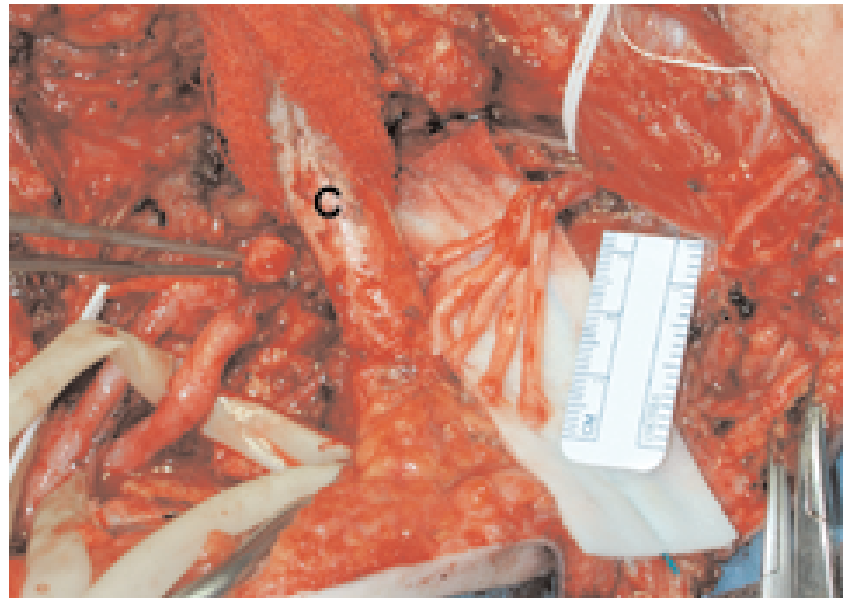

Fig. 3. Operative photograph of a contusive lesion due to a GSW. There was no NAP transmitted; thus the lesion was resected and a 3-cm interfascicular graft was placed from the C-5 spinal nerve to divisions. The distal portion of the graft repair is shown held by forceps prior to being sectioned back to healthy tissue of the lateral cord $(\mathrm{C}=$ clavicle $)$.

ments and grafts in three elements, with results of Grade 3 or better in five $(83 \%)$ of six elements and two $(67 \%)$ of three elements, respectively.

\section{Associated Injuries}

There was a high incidence of associated injuries in the LSUHSC series of GSWs involving the brachial plexus, and these injuries required relatively acute operations for repair.

The most frequent concomitant injuries were vascular injuries that were in one of two categories. The first category included injuries found at the time of acute exploration for suspected major vascular interruption. Indications for exploration included the following: 1) a penetrating wound of the neck, shoulder, or upper arm;2) diminished or absent brachial or distal radial pulses; 3) a cool hand; 4) an expanding and/or sizeable hematoma mass; 5) persistent bleeding from the wound; and 6) progressive swelling of a distal extremity due to ischemia or venous insufficiency. In most of the LSUHSC patients, angiography performed before operation had demonstrated occlusion or vascular compromise in the region of the presumed involved brachial plexus. Some wounds, however, with suspected vascular injury as well as injury to the nerves were explored acutely without preoperative angiography, usually because of persistent bleeding from the wound or an expanding hematoma. Repairs to the major arteries varied from simple suture to the use of vein or synthetic graft replacements. The status of the plexus elements had not been consistently noted when vascular repair had been performed elsewhere, whereas in a few other cases, transected elements had been "tacked down" to adjacent fascial planes. This technique appeared to be effective in maintaining the length of the neural structures. On the other hand, secondary operation for plexus repair sometimes revealed that neural elements had been incorporated by suture into the vascular repair. 
The second category of vascular injury was that of pseudoaneurysm, which was less frequent, harder to diagnose, and not easy to treat. Diagnosis was usually suspected because of the following: 1) progressive pain; 2) neural loss; 3) the presence of a thrill and bruit; and 4) a palpable mass. If angiography documented a pseudoaneurym, it most commonly revealed irregularity of the contrast column in a portion of the axillary artery, without contrast-filling of the aneurysm. At exploration, a large mass of variably organized and often encapsulated clot was found to be encircling the axillary artery and displacing the cords and cord-nerve connections of the plexus. To isolate and gain control of the proximal and distal axillary artery and its profundus brachii branch, dissection above, below, and distally was required. Vessels were then encircled with plastic loops above and below the lesion. The covering of the organized clot could then be opened after dissecting the neural elements free from its capsule. An external neurolysis was then performed on these plexus elements. Repair of the vessel was usually straightforward, that is, the vascular rent was oversewn in most instances, but occasionally had to be patched by a portion of vein. Interposition vein graft repair of the whole vessel was only rarely required.

\section{Pain Management}

Management of pain was difficult in this series of GSWs in patients treated at LSUHSC involving the brachial plexus. A few patients had true CRPS and experienced severe pain and autonomic disturbances, usually in the hand, and could not tolerate having the hand touched or manipulated. Most of these patients had received a trial of phenoxybenzamine or similar sympatholytic agents and such management usually had failed, although other authors have had greater success with such a pharmacological approach to CRPS associated with GSWs. ${ }^{2}$ Patients with true CRPS were helped by repeated sympathetic blocks alone or sympathetic blocks followed by cervical sympathectomy. Jebara and Saade $^{3}$ also documented dramatic relief with sympathectomy in their series of patients with CRPS due to brachial plexus GSWs. Patients with non-CRPS neuropathic pain required vigorous pharmacological management as well as physical and occupational therapy. Surgery on the injured plexus and manipulations of the elements with or without repair helped in some cases.

\section{DISCUSSION}

Missile injuries to the brachial plexus may be caused by low-velocity missiles, usually shell fragments that damage nerve elements by direct impact, ${ }^{12}$ and these may display a significant return of function within a few months. ${ }^{14}$ High-velocity missiles, on the other hand, have three mechanisms of nerve damage: direct impact, shock waves, and cavitation effects. Those injured by high-velocity missiles failed to display a significant return of function in our experience. ${ }^{12}$ According to Omer ${ }^{10}$ this potential for damage, however, is only realized with greater tissue penetration, that is, $12 \mathrm{~cm}$.

Older literature concerning GSWs to the brachial plex- us emphasized that many if not most cause partial neurological loss and thus would recover without surgery. The findings of complete loss in 202 (69\%) of 293 elements in the present LSUHSC series would seem to somewhat contradict the earlier literature. In the series of brachial plexus GSWs presented by Kline ${ }^{5}$ there were only 19 (21\%) of 90 patients having 166 injured elements with complete or nearly complete loss in the distribution of all elements at the level of the injury. Thus, if viewed by functional loss on the whole, most of the injuries in the current LSUHSC series do result in partial neurological loss; however, for individual elements, the functional loss is often actually complete for that individual element causing the loss of function. The ultimate functional sum is best realized by maximizing the function of the individual elements. To best maximize recovery of the individual elements, if loss in the distribution of one or more elements persists in the early postinjury months, then the patient must have an operation at which time intraoperative NAPs on lesions in continuity are performed. If a negative NAP is obtained, then lesions must be resected and repaired. Lesions found to not be in continuity are also repaired. It is essential then that clinicians evaluate each plexus injury by the elements involved and not just according to the plexus as a whole.

Intraoperative stimulation and NAP recording studies then are important to the identification of those elements needing resection. The majority of lesions in continuity with complete preoperative loss of function were treated by resection because of the absence of NAPs. On the other hand, a significant number were spared because recordings showed evidence of regeneration. Approximately $70 \%$ repaired by suture and just a little more than $50 \%$ of lesions repaired by grafts had successful outcomes.

Gunshot wounds to the brachial plexus usually result in lesions in continuity. These lesions in continuity, however, must not be assumed to recover spontaneously if treated conservatively or if only neurolysis without NAP testing is performed. Intraoperative NAPs were found to indicate lack of regeneration at 2 months postinjury in a large number of such elements in continuity. If there was complete loss in the distribution of one or more elements and the function improved, then continued conservative management was maintained.

Adult patients with lesions of the C-8, T-1, lower trunk, or medial cord to ulnar nerve were treated conservatively unless pain was not manageable by pharmacological means because repair was thought to have a low yield regarding ultimate functional recovery.

Often an associated vascular injury will warrant emergency repair. ${ }^{5,13}$ In addition to transections of a major vessel, GSWs involving the brachial plexus can produce pseudoaneurysms or arteriovenous fistulas, which can compress the plexus and produce progressive loss of function and severe pain. Injured elements need to be dissected and gently moved away from the area of vascular repair. It may also be necessary to perform a second operation for NAP recordings and neural repair.

In this series, a low incidence of serious complications (vascular, further loss of function, wound hematoma and infection) was observed, but the decision for surgical intervention must always be weighed with the knowledge that complications and poor outcomes are possible. It is 
also essential that surgery be supplemented by intensive physical therapy, lengthy follow up and monitoring, and consultation with other specialists for further reconstructive or rehabilitative measures.

\section{CONCLUSIONS}

Gunshot wounds usually leave lesions in continuity and these may cause incomplete loss of the involved element(s), which may recover spontaneously with time, although often they may not. Plexus injury should be evaluated by elements involved in addition to the plexus as a whole. Elements not in continuity should be repaired in several weeks. Operative intervention to GSWs of the brachial plexus should be delayed 2 to 4 months for the following reasons: 1 ) if there is a serious deficit with complete loss of function in one or more elements that fails to improve; 2) if there is incomplete loss that does not improve after a few months accompanied by pain of a non-CRPS nature not helped by conservative management; 3 ) if there is a pseudoaneurysm, clot, or fistula compressing or involving the plexus; and 4) if true CRPS occurs requiring sympathectomy. Intraoperative NAPs should be performed on elements in continuity and if NAPs are negative, then these lesions should be resected and repaired by suture or graft.

\section{References}

1. Caruso RP, Jara DI, Swan KG: Gunshot wounds: bullet caliber is increasing. J Trauma 46:462-465, 1999

2. Ghostine SY, Comair YG, Turner DM, et al: Phenoxybenzamine in the treatment of causalgia. Report of 40 cases. J Neurosurg 60:1263-1268, 1984
3. Jebara VA, Saade B: Causalgia: a wartime experience-report of twenty treated cases. J Trauma 27:519-524, 1987

4. Kim DH, Cho YJ, Tiel RL, et al: Outcomes of surgery in 1019 brachial plexus lesions treated at Louisiana State University Health Sciences Center. J Neurosurg 98:1005-1016, 2003

5. Kline DG: Civilian gunshot wounds to the brachial plexus. J Neurosurg 70:166-174, 1989

6. Kline DG, Hudson AR: Nerve Injuries: Operative Results for Major Injuries, Entrapments, and Tumors. Philadelphia: WB Saunders, 1995

7. Kline DG, Judice DJ: Operative management of selected brachial plexus lesions. J Neurosurg 58:631-649, 1983

8. Kline DG, Kott J, Barnes G, et al: Exploration of selected brachial plexus lesions by the posterior subscapular approach. J Neurosurg 49:872-880, 1978

9. Midha R: Epidemiology of BPIs in a multitrauma population. Neurosurgery 40:1182-1189, 1997

10. Omer GE Jr: Peripheral nerve injuries and gunshot wounds, in Omer GE, Spinner M, Van Beek AL (eds): Management of Peripheral Nerve Problems. Philadelphia: WB Saunders, 1998, pp 398-405

11. Peach SA, Ackerman C, Visser JH: Results of treatment of BPIs. J Bone Joint Surg Br 83-B (Suppl I):12, 2001 (Abstract)

12. Samardzic MM, Rasulic LG, Grujicic DM: Gunshot injuries to the brachial plexus. J Trauma 43:645-649, 1997

13. Stewart MP, Birch R: Penetrating missile injuries of the brachial plexus. J Bone Joint Surg Br 83:517-524, 2001

14. Vrettos BC, Rochkind S, Boome RS: Low velocity gunshot wounds of the brachial plexus. J Hand Surg Br 20:212-214, 1995

Manuscript received March 17, 2004.

Accepted in final form April 2, 2004.

Address reprint requests to: Daniel H. Kim, M.D., Department of Neurosurgery, Stanford University Medical Center, Room R-2001, Edwards Building, 300 Pasteur Drive, Stanford, California 943055327. email: neurokim@stanford.edu. 